\title{
Ascaridíase hepatobiliar complicada por pneumonia lipoídica
} Hepatobiliary ascariasis complicated by lipoid pneumonia

Lisieux Eyer de Jesus ${ }^{1}$, Selma Maria A. Sias², Alan Araújo Vieira³, lara Teixeira Gonçalves ${ }^{4}$, Rafael Del Castillo Villalba Marcia Regina F. André ${ }^{6}$

\section{RESUMO}

Objetivo: Apresentar um caso de ascaridíase hepatobiliar complicado por pneumonia lipoídica e discutir as implicações anestésicas envolvidas.

Descrição do caso: Menina de dois anos de idade com ascaridíase hepatobiliar complicada por pneumonia lipoídica por aspiração e desnutrição grave, advinda de família em condições sociais precárias em zona rural, com quatro irmãos. Foi tratada com sucesso por uma combinação de lavados broncopulmonares sucessivos e cirurgia.

Comentários: Ascaridíase biliar corresponde a cerca de $10 \%$ dos casos de complicações de ascaridíase. Apenas uma minoria precisa de tratamento cirúrgico. $\mathrm{O}$ uso de óleo mineral por via oral é um tratamento tradicional para a suboclusão intestinal pelo Ascaris lumbricoides, mas a broncoaspiração do óleo e a conseqüente pneumonia lipoídica representam um risco alto para o seu uso. Anestesia geral para laparotomia exploradora em pré-escolar desnutrido com pneumonia lipóide e ascaridíase biliar é uma situação pouco contemplada na literatura médica, o que exigiu um planejamento terapêutico específico.

Palavras-chave: ascaridíase; pneumonia lipóide; criança.

\section{ABSTRACT}

Objective: To present a case of hepatobiliary ascariasis complicated by exogenous lipoid aspirative pneumonia and the anesthetic implications involved.

Case description: We present a case of hepatobiliary ascariasis complicated by exogenous lipoid aspirative pneumonia and severe undernourishment in a two-year-old female from a five-children poor family from the Brazilian rural area. She was successfully treated by the association of repeated bronchopulmonary lavage and surgery.

Comments: Biliary ascariasis corresponds roughly to $10 \%$ of complicated ascariasis cases. Only a minority requires surgery. Mineral oil is a traditional treatment for intestinal Ascaris lumbricoides subocclusion, but oil aspiration and lipoid pneumonia remain a highly morbid risk of this practice. General anesthesia and laparotomy in an undernourished small child with lipoid pneumonia and biliary ascariasis were rarely addressed in the medical literature. Therefore, the therapeutic planning of this case was difficult.

Key-words: ascaridiasis; pneumonia, lipid; child.
${ }^{1}$ Titular do Colégio Brasileiro de Cirurgiões do Rio de Janeiro (CBC-RJ), titular da Sociedade Brasileira de Cirurgia Pediátrica, professor do Hospital Universitário Antônio Pedro da Universidade Federal Fluminense (Huap/ UFF), Rio de Janeiro, RJ, Brasil

2Pneumologista pediátrica e broncoscopista, professora de Pediatria do Huap/UFF, Rio de Janeiro, RJ, Brasil

${ }^{3}$ Pediatra, professor de Pediatria do Huap/UFF, Rio de Janeiro, RJ, Brasil

${ }^{4}$ Anestesiologista do Huap/UFF, Rio de Janeiro, RJ, Brasil

${ }^{5}$ Gastroenterologista pediátrico, professor de Pediatria, Huap/UFF, Rio de Janeiro, RJ, Brasil

${ }^{6}$ Residente de Pediatria do Huap/UFF, Rio de Janeiro, RJ, Brasil
Endereço para correspondência:

Lisieux Eyer de Jesus

Rua Presidente Domiciano, 52, apto. 801 - Boa Viagem CEP 24210-270 - Niterói/RJ

E-mail: lisieux@uol.com.br

Recebido em: 10/12/2007

Aprovado em: 24/2/2008 


\section{Introdução}

A ascaridíase tem alta prevalência em comunidades pobres e países tropicais. Uma minoria apresenta complicações, em especial crianças menores, desnutridas, imunossuprimidas e maciçamente infestadas, principalmente com suboclusão intestinal e ascaridíase biliopancreática ${ }^{(1,2)}$.

Mesmo os casos complicados costumam ser tratados por métodos clínicos. Poucos precisam de cirurgia ${ }^{(1-3)}$. A utilização de óleo mineral no tratamento de complicações da infestação é controversa pelo risco de broncoaspiração e indução de pneumonia lipoídica.

Assim, o objetivo deste estudo foi apresentar um caso de ascaridíase hepatobiliar complicado por pneumonia lipoídica e discutir as implicações anestésicas envolvidas.

\section{Descrição de caso}

Menina, dois anos de idade, $8,4 \mathrm{~kg}$, gravemente desnutrida, possuía quatro irmãos e condições sociais precárias, sendo moradora da zona rural. A criança foi admitida no hospital local com vômitos repetitivos, distensão e dor abdominal, diarréia e eliminação oral e anal de vários Ascaris lumbricoides há cerca de 48 horas. Apresentava desidratação leve, com perfil hidreletrolítico normal. Uma radiografia abdominal em ortostatismo sugeriu ascaridíase maciça, com vários níveis hidroaéreos em intestino delgado. Foi tratada com mebendazol, piperazina e óleo mineral oral. Cinco dias após o início do tratamento, apresentou febre e taquipnéia. Sua ausculta pulmonar mostrava estertores, sibilos e roncos difusos. Houve piora da distensão abdominal, acompanhada de hepatomegalia, sem icterícia. Os níveis séricos de aminotransferases e fosfatase alcalina estavam normais, com $7 \%$ de eosinófilos, sem neutrofilia. A radiografia de tórax mostrou várias áreas bilaterais de consolidação pulmonar. A ultra-sonografia abdominal evidenciou Ascaris lumbricoides nas vias biliares intra-hepáticas, colédoco e hepático comum e dois "abscessos" intra-hepáticos por vermes (segmentos 2 e 7). A criança foi transferida para o Hospital Universitário Antônio Pedro com o diagnóstico de pneumonia hospitalar e ascaridíase biliar.

No hospital de referência, a tomografia computadorizada de tórax mostrou áreas difusas bilaterais de consolidação pulmonar com densidade Hounsfield negativa, sugerindo pneumonia lipoídica. A broncoscopia com lavado brônquico confirmou a presença de uma substância opalescente oleosa intrabrônquica. O estudo citológico evidenciou inclusões lipídicas em macrófagos e glóbulos de gordura (coloração com Sudan 4), conforme Figura 1. Em anamnese dirigida, a mãe referiu um episódio de asfixia imediatamente depois da administração de óleo mineral.

A paciente foi tratada com antibióticos (via parenteral), nutrição enteral e lavados bronco-alveolares seriados. Não houve resolução espontânea da ascaridíase biliar em ultrasonografias seriadas e, após seis semanas, foi indicada a cirurgia. Considerando a doença pulmonar, a anestesia foi induzida e mantida por via intravenosa, sem anestésicos inalatórios. A paciente foi intubada e uma estratégia "gentil" foi aplicada para evitar barotrauma pulmonar. Laparotomia subcostal direita, colecistectomia, coledocotomia distal, lavagem e instrumentação das vias biliares (utilizando instrumental cirúrgico específico e cateteres do tipo Fogarty), debridamento e drenagem dos "abscessos" hepáticos e coledocostomia à Kehr foram executados. O colédoco e o duto hepático comum estavam espessados e dilatados. Onze Ascaris lumbricoides mortos foram removidos dos canais biliares e dos "abscessos" hepáticos. A colangiografia peri-operatória, após a instrumentação biliar, mostrou canais biliares dilatados sem vermes residuais e uma fístula intra-hepática conectando o duto hepático esquerdo ao “abscesso" do segmento 2 do fígado (Figura 2A). A paciente se recuperou plenamente da anestesia, imediatamente após o ato cirúrgico, sem necessidade de assistência ventilatória suplementar.

A criança foi submetida a um total de seis lavados brônquicos (três no pós-operatórios). Depois de três semanas de pósoperatório, nova colangiografia mostrou resolução completa da dilatação biliar e cicatrização da fístula (Figura 2B). Os drenos foram então removidos. As radiografias pulmonares

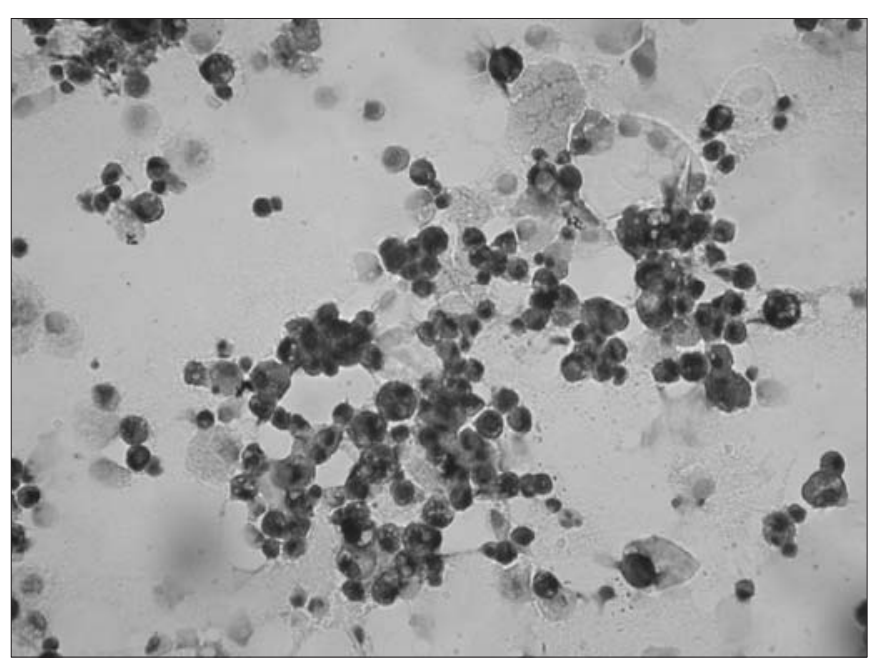

Figura 1 - Citologia do espécime obtido por broncoscopia. A coloração por Sudan 4 mostra macrófagos com inclusões. 


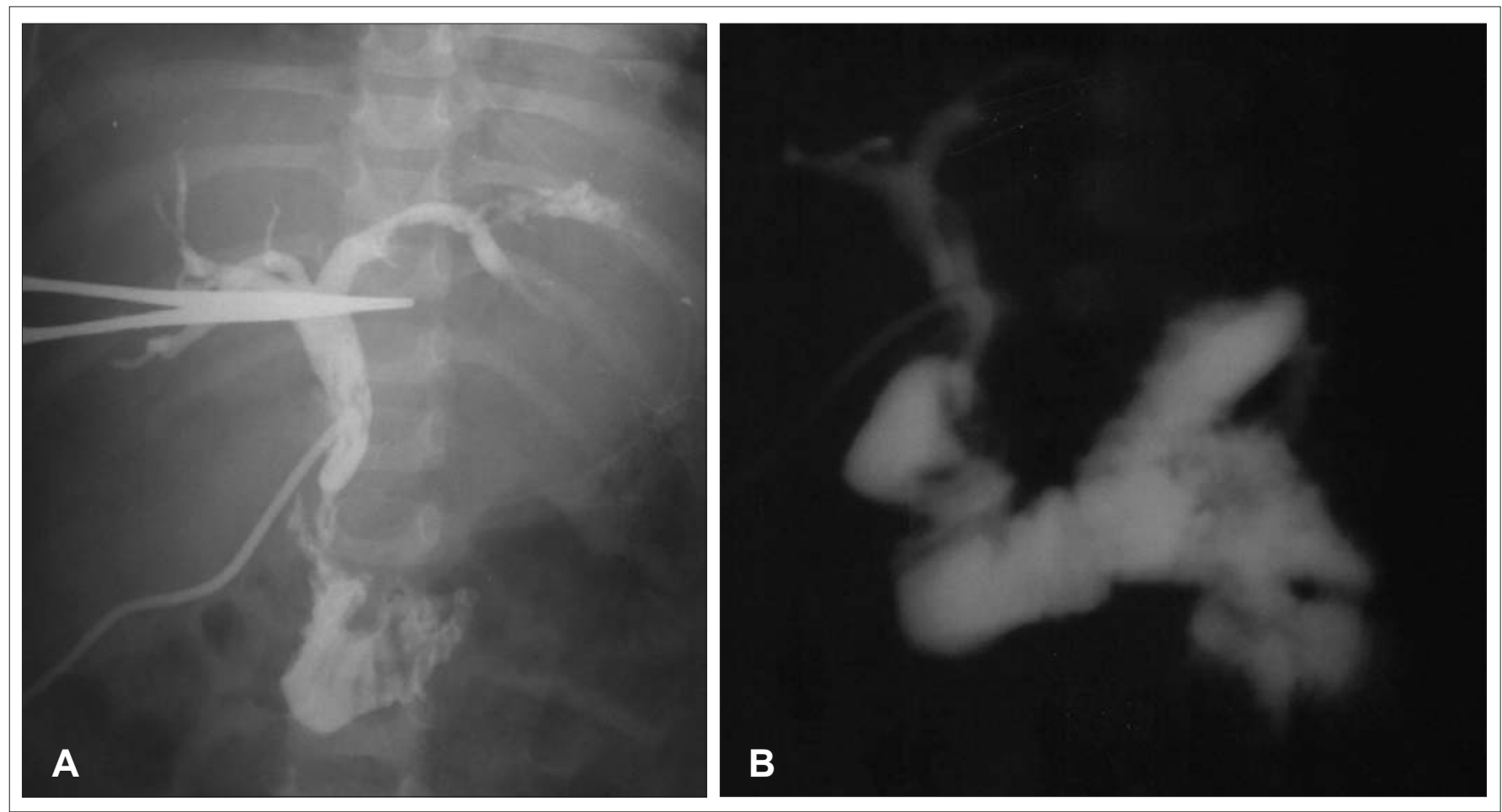

Figura 2 - (A) Colangiografia transoperatória mostrando dilatação de vias biliares e fístula do duto hepático esquerdo para o "abscesso"; (B) Colangiografia pós-operatória mostrando resolução da fístula.

confirmaram a resolução do processo pneumônico. A paciente ganhou $3 \mathrm{~kg}$ durante sua estada hospitalar e recebeu alta em estado adequado de nutrição.

\section{Discussão}

As complicações da ascaridíase são mais prováveis em infestações maciças, obviadas pela eliminação de vermes. Em áreas endêmicas, a ascaridíase causa $27 \%$ dos episódios de suboclusão intestinal e $77 \%$ dos problemas biliopancreáticos em Pediatria ${ }^{(1-2)}$. A afecção hepatobiliar é típica de pré-escolares e soma $30 \%$ dos casos de ascaridíase complicada na infância: $6 \%$ dos casos requerem intervenção cirúrgica ou endoscópica. Menos de $1 \%$ apresentarão uma massa hepática de vermes e tecido necrótico (“abscesso”) (3-4).

Os vermes nas vias biliares podem causar obstrução mecânica, espasmo do esfíncter de Oddi, inflamação e estenose. A migração biliar dos vermes está relacionada à superinfestação, à idade jovem (o pequeno calibre das vias biliares complica a apresentação e o tratamento), à imunossupressão e à desnutrição. A apresentação mais comum é a cólica biliar ${ }^{(3)}$. As formas complexas (colangite, colecistite, obstrução de vias biliares, "abscesso" hepático, colilitíase e pancreatite) são menos comuns ${ }^{(5)}$.
A ultra-sonografia é o exame de imagem preferencial para o diagnóstico de complicações biliopancreáticas da ascaridíase: os vermes são descritos como imagens lineares ecogênicas curvas ou lineares, com o interior anecóico.

A eliminação espontânea dos vermes intrabiliares depende de movimentos ativos do helminto. Em nosso paciente, foram utilizados piperazina e mebendazol. Todos os vermes retirados estavam mortos. Em nossa opinião, anti-helmínticos devem ser evitados até a saída dos vermes das vias biliares e um clareamento completo no ultra-som. Há opiniões contrárias, argumentando que as drogas não são absorvidas ou eliminadas na bile $e^{(2,5-7)}$.

Casos não complicados de ascaridíase biliar devem ser tratados clinicamente com anti-espasmódicos, com 90\% de sucesso. Recomenda-se aguardar a cura espontânea por seis semanas, monitorando-se com ultra-som a cada três a sete dias. $\mathrm{O}$ uso de antibióticos é controverso. Alguns autores advogam um período breve de antibioticoterapia pela contaminação dos vermes com a flora do intestino delgado. Dieta por via oral/enteral é utilizada mediante critérios clínicos usuais.

A extração endoscópica é preferencial, se for necessária a intervenção armada (colangite, obstrução, dor persistente, invasão biliar persistente após um período razoável de se- 
guimento - em torno de seis semanas) ${ }^{(8)}$. As desvantagens do tratamento endoscópico incluem a necessidade de anestesia geral e expertise endoscópica, equipamento adequado a crianças pequenas e papiloesfincterotomia, se for preciso extrair vermes inteiramente contidos no interior das vias biliares, o que favorece a reinfestação biliar ${ }^{(9)}$. A manipulação do colédoco é arriscada e difícil em crianças pequenas.

"Abscessos" hepáticos (por vermes e tecido necrótico, raramente contendo pus), falha do tratamento endoscópico, colecistite e perfuração biliar exigem cirurgia. O cirurgião deve sanear completamente e drenar as vias biliares. Cateteres de Fogarty são uma boa alternativa para recuperar vermes nas vias biliares estreitas do pré-escolar. Os "abscessos" são debridados e drenados. Os drenos biliares são removidos depois da colangiografia de controle demonstrar ausência de corpos estranhos intrabiliares e o fechamento de fístulas.

A pneumonia lipóide é uma circunstância única deste caso. O óleo mineral é usado tradicionalmente no tratamento de suboclusões por Ascaris lumbricoides no Brasil, embora não haja evidências de boa qualidade para defendêlo. Há riscos envolvidos, já que a bronco-aspiração do me- dicamento pode causar pneumonia lipoídica - eventualmente fatal - e o óleo mineral oral está contra-indicado em bebês com idade inferior a nove meses e em crianças com alteração de consciência ou problemas de deglutição ${ }^{(10)}$. O diagnóstico depende da história (aspiração testemunhada, tosse ou asfixia após administração), seguida por pneumonia grave, que não melhora com antibióticos e demonstração tomográfica de múltiplas áreas de consolidação pulmonar com densidade de gordura ${ }^{(11,12)}$. Ajudam no diagnóstico a remoção broncoscópica de substância gordurosa em lavado brônquico e o estudo citológico com detecção de partículas de lipídio fagocitadas pelos macrófagos pulmonares. O tratamento é controverso. Lavagens bronco-alveolares repetitivas para remover o agente agressor são aceitas porque o óleo mineral limita a ventilação e pode induzir à fibrose pulmonar ${ }^{(13)}$.

Não há estudos publicados a respeito de técnicas de anestesia em portadores de pneumonia lipoídica. A estratégia usada para este paciente visou não acentuar os problemas pulmonares, o que poderia ocorrer com anestésicos inalatórios ou ventilação sob pressão positiva.

\section{Referências bibliográficas}

1. Chang CC, Han CT. Biliary ascariasis in childhood. Chin Med J 1966;85: 167-71.

2. Malik AH, Saima BD, Wani MY. Management of hepatobiliary and pancreatic ascariasis in children of an endemic area. Ped Surg Int 2006;22:164-8.

3. Costa-Macedo LM, Costa M, Almeida LM. Ascaris lumbricoides in infants: a population-based study in Rio de Janeiro, Brasil.Cad Saude Publica 1999;15:173-8.

4. St Georgiev V. Pharmacotherapy of ascariasis. Exper Opin Pharmacother 2001;2:223-39.

5. Jesus LE, Raposo RP, Guazelli A. Ascaridíase biliar complicada: espectro de problemas e táticas cirúrgicas. Rev Col Bras Cir 2004;31:172-9.

6. Rode $\mathrm{H}$, Cullis $\mathrm{S}$, Millar A, Cremin B, Cywes S. Abdominal complications of Ascaris lumbricoides in children. Pediatr Surg Int 1990;5:397-401.

7. Vásquez Tsuji O, Gutiérrez Castrellón P, Yamazaki Nakashimada MA, Arredondo Suárez JC, Campos Riveral T, Martínez Barbosa I. Anthelmintics as a risk factor in intestinal obstruction by Ascaris lumbricoides in children. Bol Chil Parasitol 2000;55:3-7.
8. González AH, Regalado VC, Van den Ende J. Noninvasive management of Ascaris lumbricoides biliary tract migration: a prospective study in 69 patients from Ecuador. Trop Med Int Health 2001:6:146-50.

9. Khuroo MS, Zargar SA, Mahajan R. Hepatobiliary and pancreatic ascariasis in India. Lancet 1990;335:1503-6.

10. Sharif F, Crushell E, O'Driscoll K, Bourke B. Liquid paraffin: a reappraisal of its role in the treatment of constipation. Arch Dis Child 2001;85:121-4.

11. Hugosson CO, Riff EJ, Moore CC, Akhtar M, Tufenkeji HT. Lipoid pneumonia in infants: a radiological-pathological study. Pediatr Radiol 1991;21:193-7.

12. Zanetti G, Marchiori E, Gasparetto TD, Escuissato DL, Soares-Souza A Jr. Lipoid pneumonia in children following aspiration of mineral oil used in the treatment of constipation: high resolution CT findings in 17 patients. Pediatr Radiol 2007;37:1135-9.

13. Kameswaran M, Annobil SH, Benjamin B, Salim M. Bronchoscopy in lipoid pneumonia. Arch Dis Child 1992;67:1376-7. 